\title{
MEASUREMENT OF RIDER INDUCED LOADS DURING SIMUILATED BICYCLING
}

Farhad Bolourchi

Department of Mechanical Engineering

University of California

Davis, CA 95616

M. L. Hull

Department of Mechanical Engineering

University of California

Davis, CA 95616

Research related to bicycling has broadened in scope over the last decade. Prior to about 1975, the majority of bicycling related research was dedicated to topics surrounding the physiology of human performance. These early efforts served to stimulate interest in bicycling research with the result that more recent research has explored a diversity of topics ranging from fundamentals of muscle mechanics to measurement of foot/pedal loads. Despite the both broadened and intensified research activity, one topic, which has received no attention to the authors' knowledge, is measurement of the complete loading induced by the rider on the bicycle frame. The importance of this topic lies in the applicability of the results to two areas, design analysis of bicycle components including the frame and biomechanical analysis of the pedalling process. The concern in the present article is with the biomechanical analysis.

Seeking to understand the biomechanics of the pedalling process, a number of researchers have recognized that pedal loading merits study. The earliest modern measurement of pedal loading is the work of Hoes et al. (1968) who used strain gages to monitor both the normal pedal force and the crank torque. Results showed that the normal pedal force is maximum when the crankarm is horizontal (i.e. $90^{\circ}$ ) during the downstroke and that the passive leg is lifted partly by the active leg.

Building on the work of Hoes et al. (1968), Gregor (1976) used a pedal dynamometer system which simultaneously measured both normal and tangential pedal forces' developed on both pedals. Depending on the subject it was found that pedal forces may be asymmetrical with the right leg developing larger forces than the left. Gregor (1976) also studied the relationship between pedal forces and workload at constant RPM and found not surprisingly that the maximum magnitude of the normal pedal force is directly related to work load.

Soden and Adeyefa (1979) undertook a comprehensive study of in-plane rider induced loading by estimating pedal loads based on power output and computing both seat and handlebar maximum loads assuming static equilibrium. Estimated pedal loads were subsequently verified by laboratory measurements using a 2-load component dynamometer. Forces were analyzed under conditions (e.g. hill climbing) likely to lead to extreme levels. Maximum normal pedal forces were computed to be twice body weight. Because maximum forces were developed when riders were standing, handlebar forces were also large reaching levels greater than body weight when the rider pulled upwards. 
Hull and Davis (1981a) extended the pedal load measurement system of both Gregor (1976) and Soden and Adeyefa (1979) by constructing a six load component dynamometer to measure the complete pedal loading. Also, the absolute spatial position of the pedal was measured using potentiometers. Davis and Hull (1981b) found that the out-of-plane loads (i.e. transverse shear, axial moment, varus-valgus moment) were significant. The transverse shear, for example, exceeded lOON with the peak value coinciding with the peak value of the normal force which occurred at a crank arm angle of $100^{\circ}$. Davis and Hull (1981b) also confirmed the direct relation between workload and pedal force reported by Gregor (1976).

while these previous research efforts have provided valuable insight into the pedalling process, a biomechanical analysis, which includes measurement of both seat and handlebar loading in addition to pedal loading has not been attempted. Therefore, one objective of this paper is to present an analysis of bicycling biomechanics where all rider induced loads are included. Inasmuchas both Gregor (1976) and Davis and Hull (1981b) restricted their attention to the relation between pedal load and workload at constant RPM, a second objective is to examine the relation between rider induced loads and RPM at constant workload.

NOMENCLATURE

$x, y, z$ Local coordinate system of the pedal, handlebar and seat dynamometers

A load is designated by at most four parameters; location, type, direction, and the side of the body by which it is being applied.

location $\quad P$ for pedal

$H$ for handlebar

$S$ for seat

type $\quad F$ for force

M for moment

direction $\quad x, y, z$

side $\quad 1$ left side

$r$ right side

examples: $H F x r=h$ andlebar force applied by the right hand in the local $x$ direction

SMy $=$ Seat post moment applied in the local y direction

$\{\mathrm{V}\}$

$\{F\}$

vector of Wheatstone bridge output voltages

vector of applied loads

[C] pedal calibration matrix

$[C]^{-1} \quad$ pedal sensitivity matrix

$\theta_{r}, \theta_{1}$ right and left pedal angles respectively referred to horizonta? 


$\begin{array}{ll}\text { W } & \text { weight of test subject } \\ \mathrm{RR} & \text { rolling resistance } \\ P & \text { tire pressure } \\ F_{n} & \text { instantaneous power } \\ \theta_{1} & \begin{array}{l}\text { component of pedal force normal to the crank arm } \\ \text { positive clockwise }\end{array} \\ \theta_{2} & \begin{array}{l}\text { absolute pedal angle with zero referenced to horizontal and } \\ \text { positive clockwise }\end{array} \\ L_{2} & \text { crank arm length } \\ \Omega & \text { crank arm angular velocity } \\ T_{C} & \text { crank arm torque }\end{array}$

METHOOS

Figure 1 shows the schematic ot the process used to obtain the final results. First analog signals are generated from the transducers (pedal, handlebar, and seatpost dynamometer plus pedal and crank potentiometers). Then the signals go through a signal conditioning phase where they are amplified. Next, signals are digitized using a computer-based analoy-to-digital converter. Once digitized, data are processed by specialized software with the main tasks being to both calibrate the digitized numbers to meaningful quantities like force, moment, and angles and plot the loading profiles. Each of these steps will be discussed in more detail.

The dynamometry requirement may be considerably simplified by considering the nature of the loading at the five body contact points, pedals, handlebars, and seat. The pedals will be discussed first. Inasmuchas Davis and Hull, (1981b) have shown that loads out of the plane of the bicycle may be significant, the pedal dynamometer must measure the six load components (see Fig. 2). The work of Gregor, (1976) however, has shown that the pedal loading is approximately symmetrical for the majority of test subjects. Accordingly, symmetry in the pedal loading can be assumed so that only one pedal dynamometer is required. Under this assumption, the loads on the right side are equal to the loads on the left side with a $180^{\circ}$ phase shift. The symmetry assumption can also be applied to the handlebars thereby necessitating only one of the bars to be instrumented.

Further simplification in both the seat and handlebar dynamometers stems from recognizing that some of the loads are insignificant. Handlebar loads, HFy, HMx, HMy, and HMz (see Fig. 4) are not applied at all unless the rider intentionally applies them. Likewise, the seat loads SFy and SMz (see Fig. 5) are not significant because it is difficult to maintain balance while applying these loads. Seat load SFx will not be measured here and, as will be shown later, it is calculated. In light of these observations a total of 11 load components ( 6 pedal, 2 handlebar, and 3 seat) need to be measured as well as the two reference angles. 

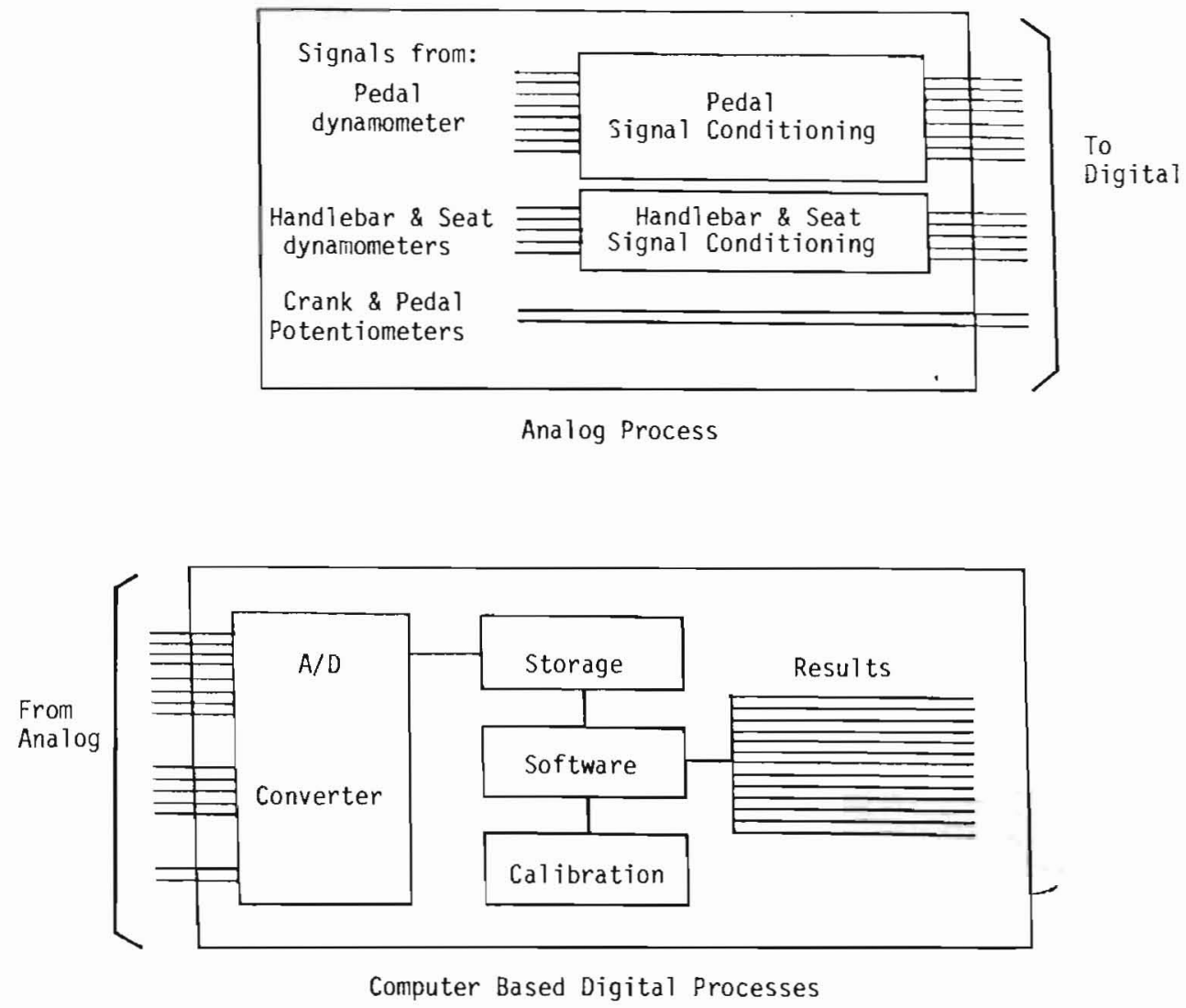

Figure 1. Schematic of data acquisition and signal processing

Three dynamometers measure the different forces and moments. One is the pedal dynamometer which measures the six loads applied to the pedal. This pedal is the same as that developed by Hull. and Davis, (1981a). Briefly, the pedal dynamometer consists of four strain rings. Eight strain gages mounted on each ring are connected into two full wheatstone bridge circuits which develop independent signals indicating the two forces normal to the axis of the ring. By combining the eight dynamometer signals (4 rings $\times 2$ signals/ring) through different arithmetic operations, it is possible to form six signals each one of which directly indicates one of the six loads developed between the foot and pedal. The coordinate system defining the orientation of pedal loads is illustrated in Fig. 2 while tne pedal dynamometer is pictured in Fig. 3. Further details of the pedal dynamometer design may be found in Hull and Davis, (1981a).

Another is the handlebar dynamometer which measures the horizontal and vertical forces applied to the handlebar. Here, four strain gages are 


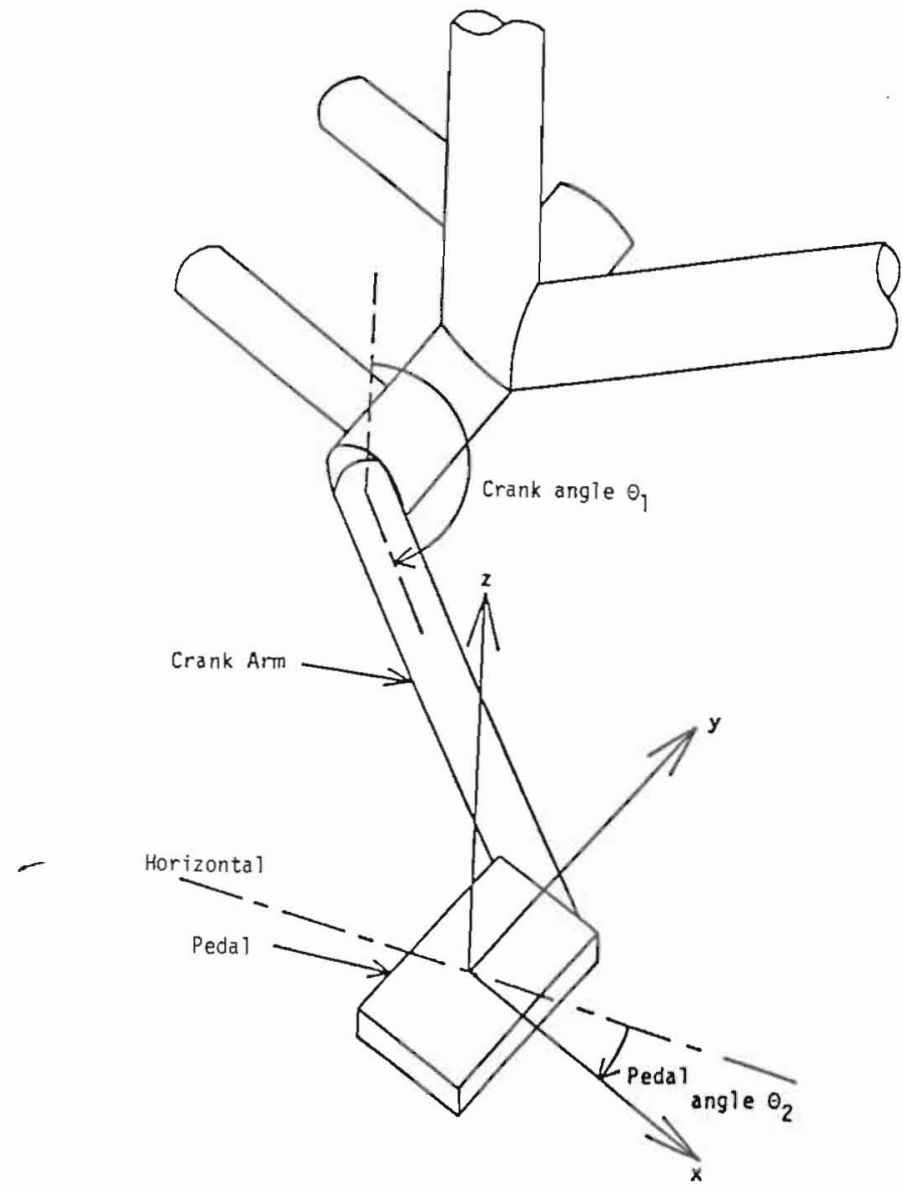

Figure 2. Definition of the local coordinate system for the pedal dynamometer ( $x-y$ Ties in the plane of the pedal)

mounted directly to the handlebar circumferentially $90^{\circ}$ apart. Gages are located close to the stem in order to produce as much sensitivity as possible. Each force is measured by a half bridge formed by a gage pair $130^{\circ}$ opposed. Note that there is minimal cross sensitivity between the two half bridges because each one is located on the neutral axis of bending created by the other force.

The third is the seat post dynamometer (see Fig. 5) which also relies on strain gages to measure SFz, SMx, and SMy. The two moments are sensed by two half bridges while the axial force is sensed by a full bridge in order to develop improved sensitivity. Again, strain gages are located and bridges are interconnected so as to minimize cross sensitivity.

The remaining transducers are the two potentiometers that measure the crank angle and the relative pedal angle (see Fig. 2). The potentiometers are single turn and continuous. Once in every revolution however, distortion occurs near the transition point. The duration of the 
transition region is about $20^{\circ}$. Special software replaces data in this region with linearly interpolated values. Zero angle references are measured using the computer prior to the actual testing. Bubble levels and other alignment tools are used to accurately position the pedal. Reference values are taken when the pedal is at Bottom Dead Center (BDC). These values are used in the post processing.

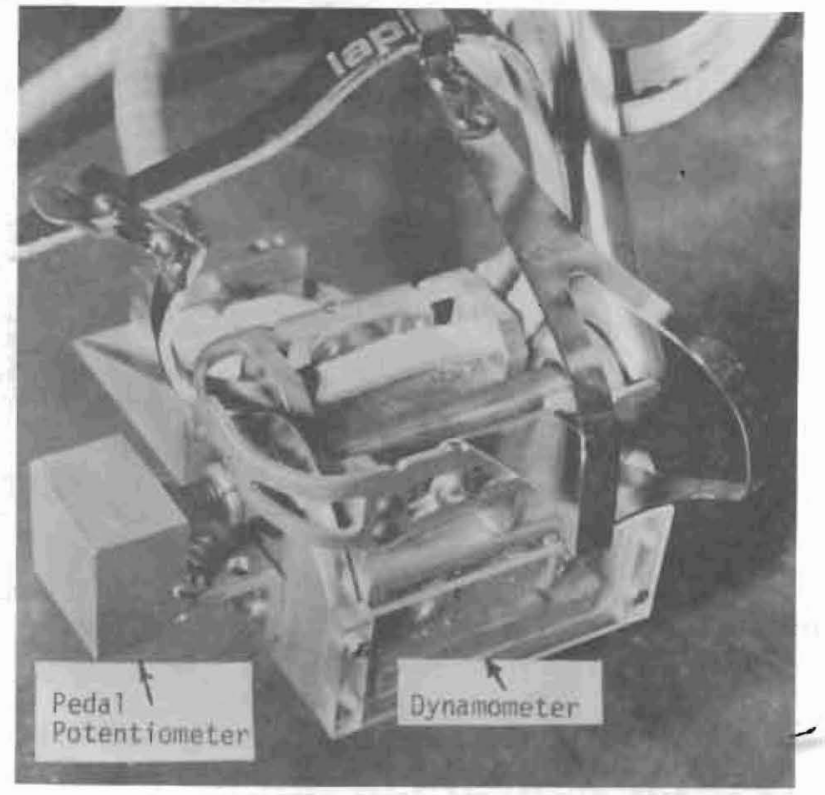

Figure 3. The 6 load component pedal dynamometer developed by Hull and Davis, (1981a).

To achieve high measurement accuracy and maintain the resolution afforded by the $A / D$ converter, the most important signal conditioning performance considerations are low drift voltage and high signal/noise ratio. Carrier amplification systems were chosen to meet these criteria effectively.

The amplified signals are digitized and stored by an LSI-11/23 computer. As configured, the computer is a powerful data collection and analysis facility. For digital data acquisition, the system features a 16 channel multiplexer and a 12 bit bipolar A/D converter. The operating system resides on a $10 \mathrm{M}$ byte disk. To view the results of data analysis, the system offers graphics capability which is supported by extensive software.

In calibration of dynamometers, the objective is to generate the coefficients of matrix [C] where

$$
\begin{aligned}
& n \times 1 \\
& \{V\}=[C] \times\{F\}
\end{aligned} \quad \begin{aligned}
& n \times n \\
& n \times 1
\end{aligned} \quad(\text { where } n \leq 6)
$$




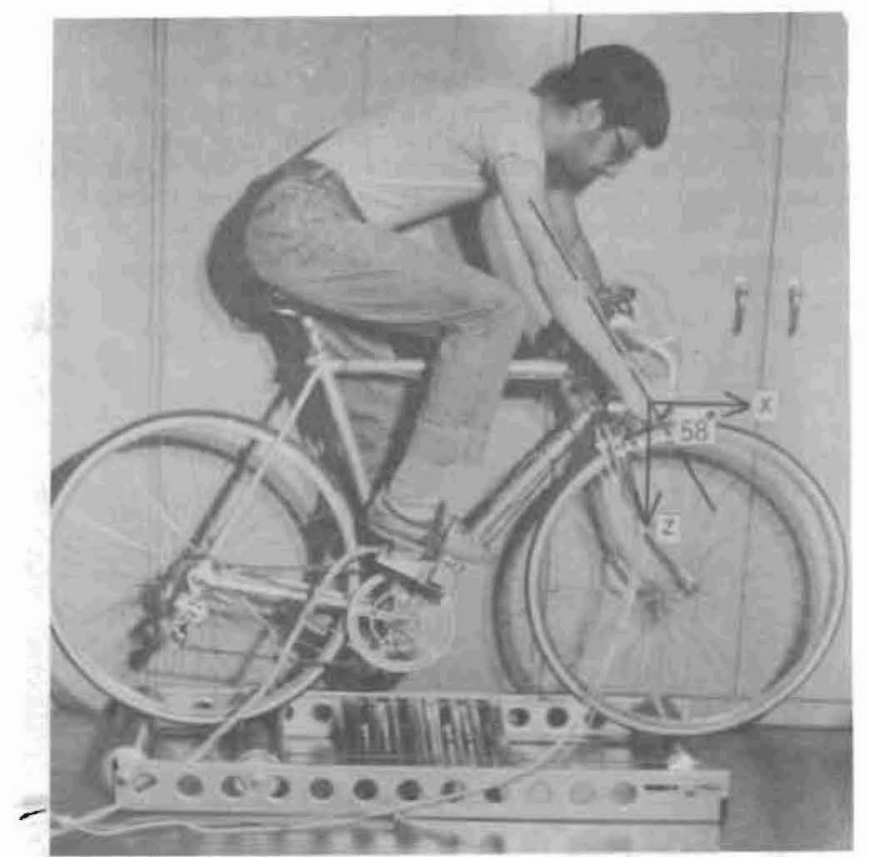

Figure 4. Definition of the local coordinate system for the handlebar dynamometer. $(x-z$ lies in the plane of the bicycle).

In Eq. (1) $\{\mathrm{V}\}$ is the vector of output voltages and $\{F\}$ is the applied load vector in units of $\mathrm{N}$ and $\mathrm{Nm}$. The calibration coefficient matrix [C] is comprised of both direct and cross-sensitive coefficients in units of $V / N$ and $V / N m$. For example, in Eq. (1) when $n=6$ the second equation becomes:

$$
V_{y}=C_{21} F_{x}+C_{22} F_{y}+C_{23} F_{z}+C_{24} M_{x}+C_{25} M_{y}+C_{26} M_{z}
$$

where $\mathrm{C}_{22}$ is the direct sensitivity and the remaining coefficients are the cross-sensitive coefficients. The cross coefficients are due either to imperfections in the transducer itself (e.g. strain gage misalignment and strain gage cross sensitivity) or to the geometric orientation of the load application point relative to the strain gage position (i,e. the moment ann) which may induce large cross coefficients.

In order to find the calibration coefficients, only one load is applied at a time (e.g. $\left.F_{X}\right)$. Then, because all other loads are zero, Eq. (2) reduces to:

$$
V_{y}=C_{21} F_{x} \text { or } C_{21}=\frac{V_{y}}{F_{x}}
$$

The applied load $F_{X}$ is incremented and $C_{21}$ is calculated from the slope of the best fit line. But Eq. (2) was only one of the six equations. So, by the same process, we can calculate $C_{i l}, i=1,6$ or the first column of the [C] matrix. By repeating this procedure for all the loads we generate [C] in its entirety. 


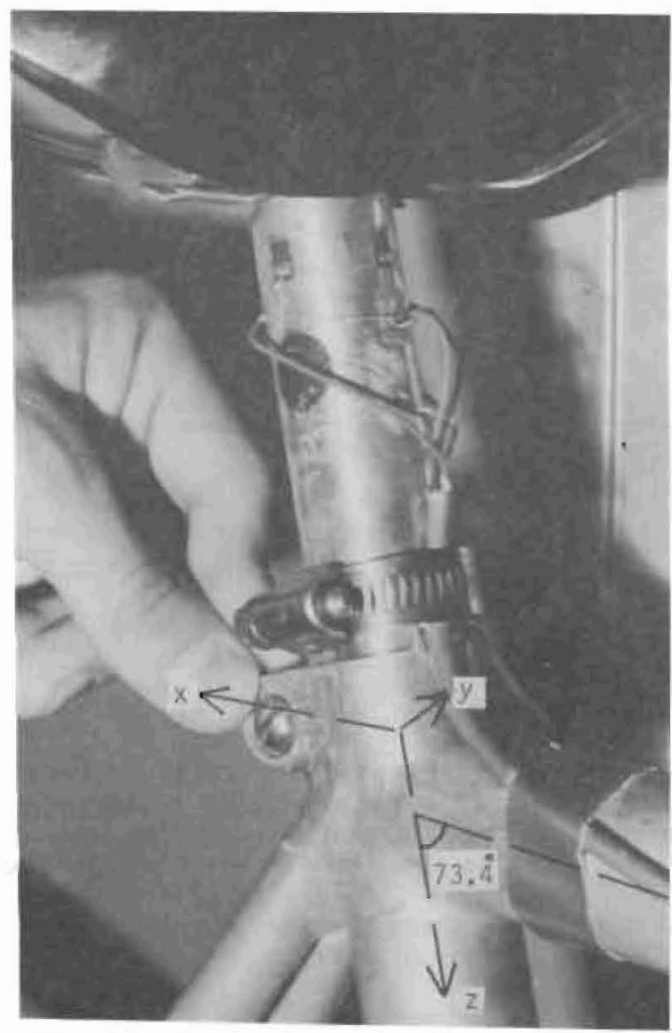

Figure 5. The three load component seat dynamometer and definition of the local coordinate system ( $z$ is along the seat tube which forms an angle of $73.4^{\circ}$ with respect to horizontal; $x-z$ Ties in the plane of the bicycle.

Uitimately when the experiments are run, it is necessary to compute the applied load vector $\{F\}$ from the output voltage vector $\{\mathrm{V}\}$. This requires that $[C]-1$ (the sensitivity matrix) be formed. The unknown loads may then readily be solved for from $\{F\}=[C]^{-1}\{V\}$. Note that while this equation must be solved at each data point, the costly computation of $[C]^{-1}$ is done only once.

A special calibration apparatus was used to calibrate the pedal dynamometer (see Hull and Davis, 1981 a for further details). Using a system of cables, weights, and pulleys, loads are applied individually to the dynamometer. Placement of weights on appropriate cables makes both positive and negative loads possible. After applying all six loads the calibration matrix was formed and was inverted in order to obtain the sensitivity matrix.

As discussed previously all cross coefficients related to the seat and handlebar dynamometers are negligible. Accordingly the calibration procedure involves finding the direct sensitive calibration coefficients, inverting them, and then performing scalar multiplication in the post processing. 


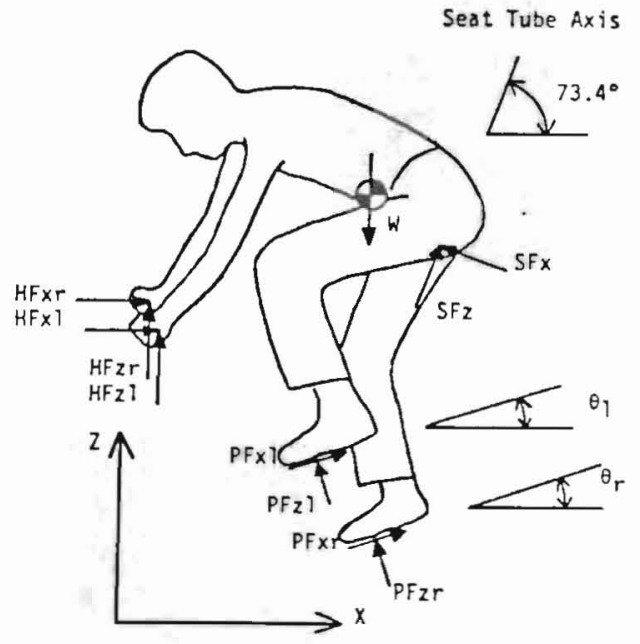

Figure 6. Freebody diagram of the rider

Laboratory experiments were executed for three different test subjects (see Table I) with each subject performing three different cases (see Table II). Because these cases all have a constant average power ( $100 \mathrm{~W} /$ pedal), the effect of different cycling RPMs can be studied along with the motive force. Throughout the experiments tire pressures are maintained at 100 psi so that rolling resistance losses are the same for all cases.

\section{CALCULATEO NEICKT}

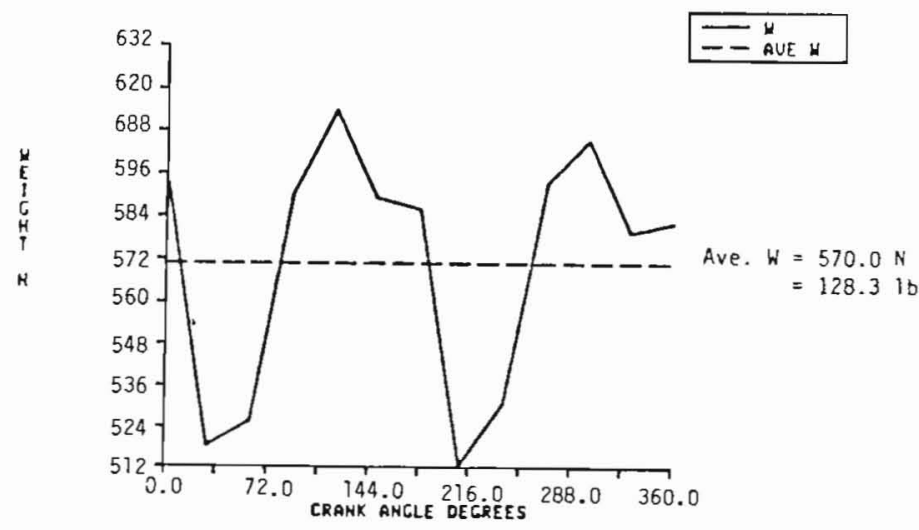

Figure 7. Calculated rider's weight 
Table I. Test Subject Information

\begin{tabular}{llll}
\hline Rider & \multicolumn{1}{c}{ Weight } & Height & Riding Experience \\
\hline A & $578.27 \mathrm{~N}(130 \mathrm{lb})$ & $1.78 \mathrm{~m}\left(5^{\prime} 10^{\prime \prime}\right)$ & 5 year local racer \\
B & $547.13 \mathrm{~N}(123 \mathrm{lb})$ & $1.66 \mathrm{~m}\left(5^{\prime} 5 \mathrm{~L}^{\prime \prime}\right)$ & 3 year local racer \\
C & $733.96 \mathrm{~N}(165 \mathrm{lb})$ & $1.83 \mathrm{~m}\left(6^{\prime} 0^{\prime \prime}\right)$ & casual rider \\
\hline
\end{tabular}

Table II. Riding Conditions

\begin{tabular}{ccc}
\hline Cases & RPM & Gear Ratio \\
\hline 1 & 63 & $52 \times 15$ \\
2 & 80 & $52 \times 19$ \\
3 & 97 & $52 \times 24$ \\
\hline
\end{tabular}

Each experiment begins with the computer operator triggering the interactive acquisition program. First the static offset voltages of the dynamometers are recorded. Second, the cycling RPM is input so that the computer can calculate the sampling frequency of quantization such that at least 360 points are digitized in each cycle. Then the riding begins with test subjects wearing lightweight shoes with cleats and toe clips. Because the riding is done on rollers (see Fig. 4) where both wheels revolve and no lateral support is provided, the subjects must balance their bicycles just as if they were riding normally. When the appropriate cadence and steadiness are obtained, the operator invokes the data acquisition routine. Once triggered, acquisition begins at the beginning of the next stroke (pedal "Bottom Dead Center" - BDC), and the raw data is taken over three full revolutions.

Data analysis is facilitated by special software which performs the following functions:

i) a - Checks for bad data (i.e. wrong cadence, data not processing through three revolutions, etc.)

b - Calculates both pedal* and crank angles by averaging the data over the three revolutions and using previously measured calibration references.

ii) Calculates the pedal, handlebar and seat loads by averaging over the three revolutions and incorporating the sensitivity matrix and coefficients. 
iii) a - Digitally filters the results to eliminate noise

b - Converts the results back to the "Top Dead Center" (TDC) reference in order to be consistant with the usual bicycling data presentation.

iv) Plots all the loads and the pedal angle vs. the crank angle.

\section{RESULTS AND DISCUSSION}

because test subject $A$ elicited what are considered to be typical results, measured quantities for this subject are presented in Figs. 8-12. Before discussing these results in detail, it is useful to perform a simple test on the relative magnitude of the forces. Because the forces are applied by the body, the sum of the components in the vertical direction must balance the rider's weight (see Fig. 6). Also, in the horizontal direction, there should be no net force on the rider because the rider is stationary and pedals at a constant rate. Applying these two equilibrium conditions and assuming that all the left side forces and angles are equal to the corresponding right side values with a $180^{\circ}$ phase difference yields:

Horizontal: PFxr $\cos \left(\theta_{r}\right)-\operatorname{PFzr} \sin \left(\theta_{r}\right)+P F \times 1 \cos \left(\theta_{1}\right)-$

$P F z l \sin \left(\theta_{1}\right)+H F x r+H f x l+S F z \cos \left(73.40^{\circ}\right)-$

SFx $\sin \left(73.40^{\circ}\right)=0$

Vertical: $\quad P F-x r \sin \left(\theta_{r}\right)+P F z r \cos \left(\theta_{r}\right)+P F x 1 \sin \left(\theta_{1}\right)+P F z l \cos \left(\theta_{1}\right)+$

$$
H F z r+H F z l+S F z \sin \left(73.40^{\circ}\right)+\operatorname{SFx} \cos \left(73.40^{\circ}\right)=W
$$

In these equations the unknowns are SFx and $W$. From Eq. (4) we solve for SFX and substitute into Eq. (5) to get $W$. Calculating $W$ based on the above equations at $30^{\circ}$ intervals gives the results shown in Fig. 7 . As can be seen, the calculated average weight during one cycle agrees almost perfectly with the actual rider's weight (see Table I). The small fluctuations ( \pm 45 $N$ ) from this average value are due to the body movements that occur during each cycle.

\section{A. Pedal Loads}

The complete pedal load profiles for rider A are presented in both Fig. 8 , which depicts the three force profiles, and Fig. 9 which depicts the three moment profiles. The general features of a typical load profile may be gleaned from examining Figs. 8 and 9 . Features, which are independent of both rider and pedalling rate, will be discussed first. Perhaps the most striking feature of the plots is the shape of the normal force curve $\mathrm{PFz}$. The force always reaches its maximum magnitude during the downstroke in the range $90^{\circ}-110^{\circ}$ and then approaches zero during the upstroke part of the cycle. The downstroke region is defined to range from $0^{\circ}-180^{\circ}$ while the upstroke region occupies the range $180^{\circ}-360^{\circ}$. Although the normal pedal force approaches zero during the upstroke, it remains negative indicating that the pedal is aiding the muscle in lifting the leg. Of course the "aid" is being provided by the opposite leg. Both the transverse pedal force PFy and axial moment PMz are always in phase with the normal pedal force PFz. As the rider extends both the hip and knee joints, the downward force on the pedal is accompanied by an outward force as well as by a tendency for the leg to twist internally. Note that maximum magnitudes of both $\mathrm{PMz}(5-10 \mathrm{Nm})$ and PFy ( 30N) are not insignificant as is usually assumed in pedal loading studies. 


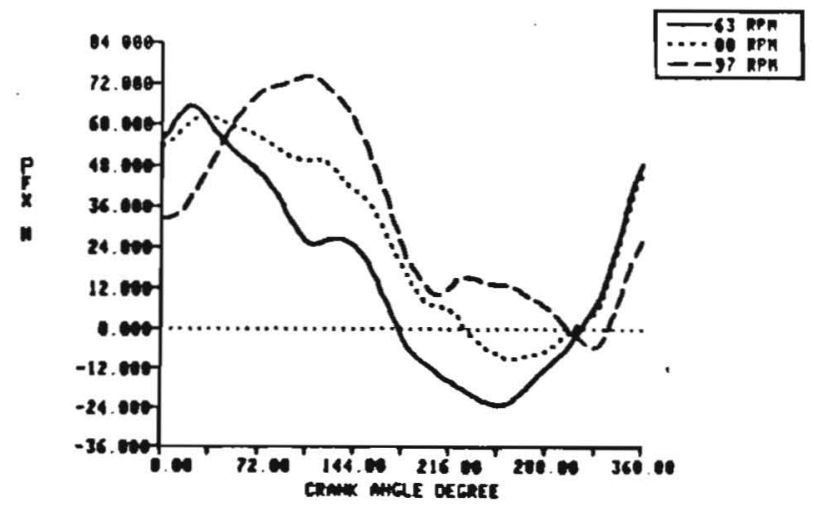

Figure 8. Pedal force profiles for rider A

The profile of the force PFx is typical when cleats with toeclips are used. Positive shear (forward) occurs during the first part of the stroke. As the pedal moves downward, the shear load shifts to a negative value during the upstroke. Then, as the pedal nears the top of the stroke, PFx becomes positive again. It is interesting to note that if the PFx profile were inverted it would nearly match the PMy profile. It may seem surprising that PMy is not always zero because the pedal platform is fixed to the spindle with low friction ball bearings. Keep in mind, however, that the force PFX is applied above the pedal spindle. The PMy moment is a necessary by-product of the shear force PFx because the total moment about the pedal spindle must be nearly zero. Accordingly, it is observed that the neyative of the PMy moment tracks the PFx force.

The most unpredictable load component is the moment PMx. PMx is generated whenever the point of application of $P F Z$ moves laterally from the center of the pedal. Such motion depends on several highly variable factors, such as lateral constraint of the foot and condition of the sho

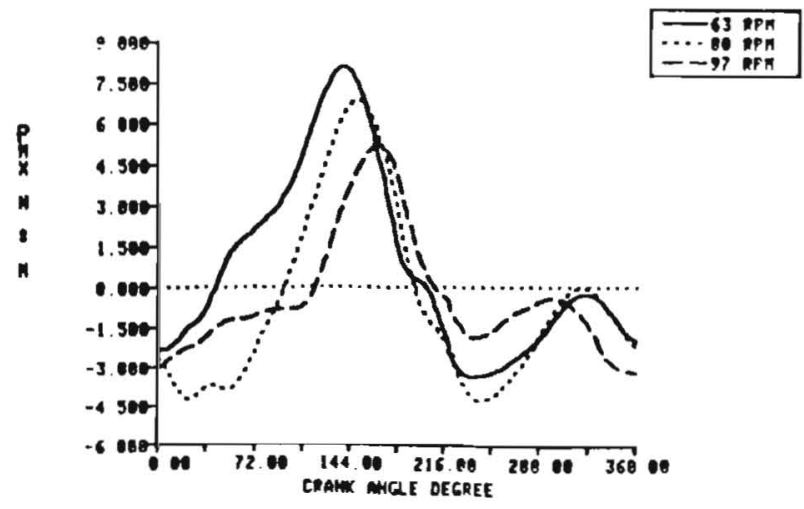

Figure 9. Pedal moment profiles for rider 
cleat grooves. Therefore, large variations in PMx profiles are observed among riders.

The crank arm torque plots companion to the pedal force plots of Fig. 8 are illustrated in Fig. 10. The total crank arm torque $T_{C}$, which acts about the pedal spindle, is given by

$$
T_{C}=F_{n} L_{2}
$$

where $L_{2}$ is the crank arm length and $F_{n}$ is the component of the resultant pedal force vector driving the crank. The pedal force component $F_{n}$ driving the crank is related to the measured pedal forces by

$$
F_{n}=P F_{z} \sin \left(\theta_{1}-\theta_{2}\right)+P F_{x} \cos \left(\theta_{1}-\theta_{2}\right)
$$

where $\theta_{1}$ and $\theta_{2}$ are the absolute crank and pedal angles referred to vertical and horizontal respectively with positive being clockwise. Figure 10 plots not only the total crank arm torque given by $E q$. (6) but also the contributions to the total torque by the normal and tangential pedal forces as indicated by Eq. (7).

Examining first the torque produced by the tangential pedal force it is noted that this force produces positive torque over two different regions. The tangential pedal force PFx contributes positive torque both in the region near TDC and in the region around $250^{\circ}$. The positive torque in the region around $T D C$ is a result of the positive tangential load in this region (see Fig. 8) while the positive torque at $250^{\circ}$ results from the negative tangential load. Negative tangential loads during the upstroke are enabled by the cleats fixed to the bottom of the shoe soles. With the pedal toeclip strap pulled taut, the cleats engage the pedal cage so that the foot cannot be extracted from the pedal.

In contrast to the tangential pedal force, positive torque resulting from the normal pedal force PFz occurs over a single broad region which ranges from about $T D C$ to $180^{\circ}$. The peak value occurs at about $100^{\circ}$ which corresponds to the crank arm being just past horizontal. Note that the positive torque in the downstroke region is enabled by the nomal pedal load being negative (see Fig. 8 ) and that the peak torque at $100^{\circ}$ coincides with the peak negative normal pedal force. At $180^{\circ}$, the torque due to the normal pedal force shifts polarity becoming negative. During the upstroke the pedal is being lifted and the negative normal pedal force (see Fig. 8) accounts for the negative torque.

Because the magnitude of the peak torque from the normal pedal force PFz is significantly greater than the magnitude of the peak torque from the tangential pedal force PFx, the shape of the total torque profile in Fig. 10 resembles that of the torque profile corresponding to PFz. The influence of the torque due to the tangential pedal force is apparent, however. Both the positive total torque at TDC and a reduction (compared to the torque due to $P F Z$ ) in the magnitude of the negative torque in the total torque profile during the upstroke are attributed to the influence of the tangential pedal force. 

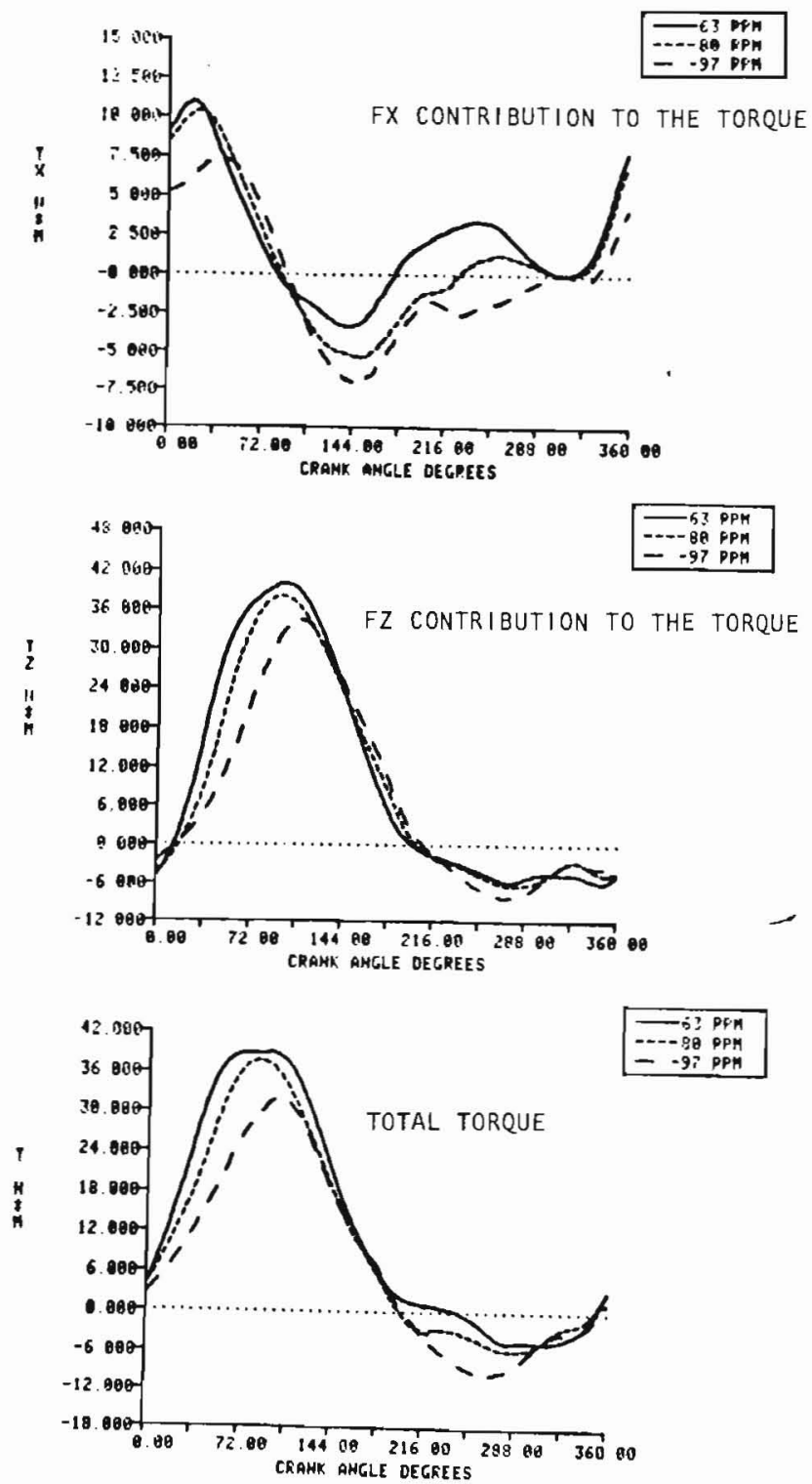

Figure 10. Crank torque profiles for rider A 
A number of features. of the pedal load profiles are related to the pedalling rate but are subject independent. First, there is a definite relation between the driving loads $P F X$ and $P F z$ and pedaliing rate. The relation depends on the region of the crank cycle, however. In considering the vertical pedal force PFz during the downstroke note that the maximum magnitude, which occurs between $90^{\circ}-110^{\circ}$, is inversely related to pedalling rate. During the upstroke, however, the magnitude of $\mathrm{PFz}$ bears a direct relation to pedaliing rate. Contrasted to $P F Z$ is the tangential pedal force $\mathrm{PFX}$ which bears an inverse relation to pedalling rate in the upstroke region especially in the vicinity of $250^{\circ}$. The relation is described as inverse because the trend towards more positive tangential load in this region leads to a decrease in torque. Although the data for rider $A$ indicate a direct relation between the magnitude of $P F x$ and pedalling rate during the second half of the downstroke $\left(90^{\circ}-180^{\circ}\right)$ this observation cannot be generalized because it does not hold for the other riders. Inasmuchas the moment PMy equilibrates the moment developed by PFX about the pedal spindle, the PMy moments exhibits characteristics similar to PFx.

To understand the inverse relations between the driving loads and pedalling rate, note that the instantaneous power $P$ is given by

$$
\text { - } \quad P=T_{C} \Omega=F_{n} L_{2} \Omega
$$

where $\Omega$ is the crank arm angular velocity. Accordingly, an inverse relation is expected during the regions in which the driving forces develop positive torque. For the normal pedal force $P F_{Z}$, where positive torque occurs throughout the downstroke, the inverse relation holds. For the tangential pedal force PFX, where positive torque occurs both at TDC and during the upstroke at about $250^{\circ}$, the inverse relation is generally valid during the upstroke but not at $T D C$. The effects of the inverse relation between the magnitude of the driving pedal forces and pedalling rate on the torque can be clearly seen in Fig. 10. The torque due to PFx is decreased over the majority of the upstroke as pedalling rate increases while the torque due to $\mathrm{PFZ}$ is decreased over the majority of the downstroke with increased pedalling rate.

The direct relation between the magnitude of the normal pedal force and the RPM during the upstroke (see Fig. 8) is interesting. The increase in magnitude is due to the pedal supporting a greater percentage of the leg weight. It appears that at higher pedaliing rates, the neuromuscular control system may not have adequate time to respond to the change in load demand (i.e. extensive to flexive) required of the leg muscles. As might be expected, the increase in magnitude of negative PFz during the upstroke has a detrimental effect on the torque (see Fig. 10). Namely, the magnitude of the negative torque contributed by $\mathrm{PFz}$ increases.

Although the effects of pedalling rate are evidenced primarily in magnitude changes of pedal load profiles, at least one load profile, namely PFX, undergoes a consistent temporal change. As illustrated in Fig. 8 , the positive peak of the tangential pedal force shifts from just after TDC for the low pedalling rate to just after $90^{\circ}$ for the high pedaliing rate. Inasmuchas the pedal angle is such that the tangential pedal force does not contribute to the crank torque at $90^{\circ}$ (see Fig. 10), the effect of this shift is to reduce the positive torque developed by $\mathrm{PFx}$ in the region 

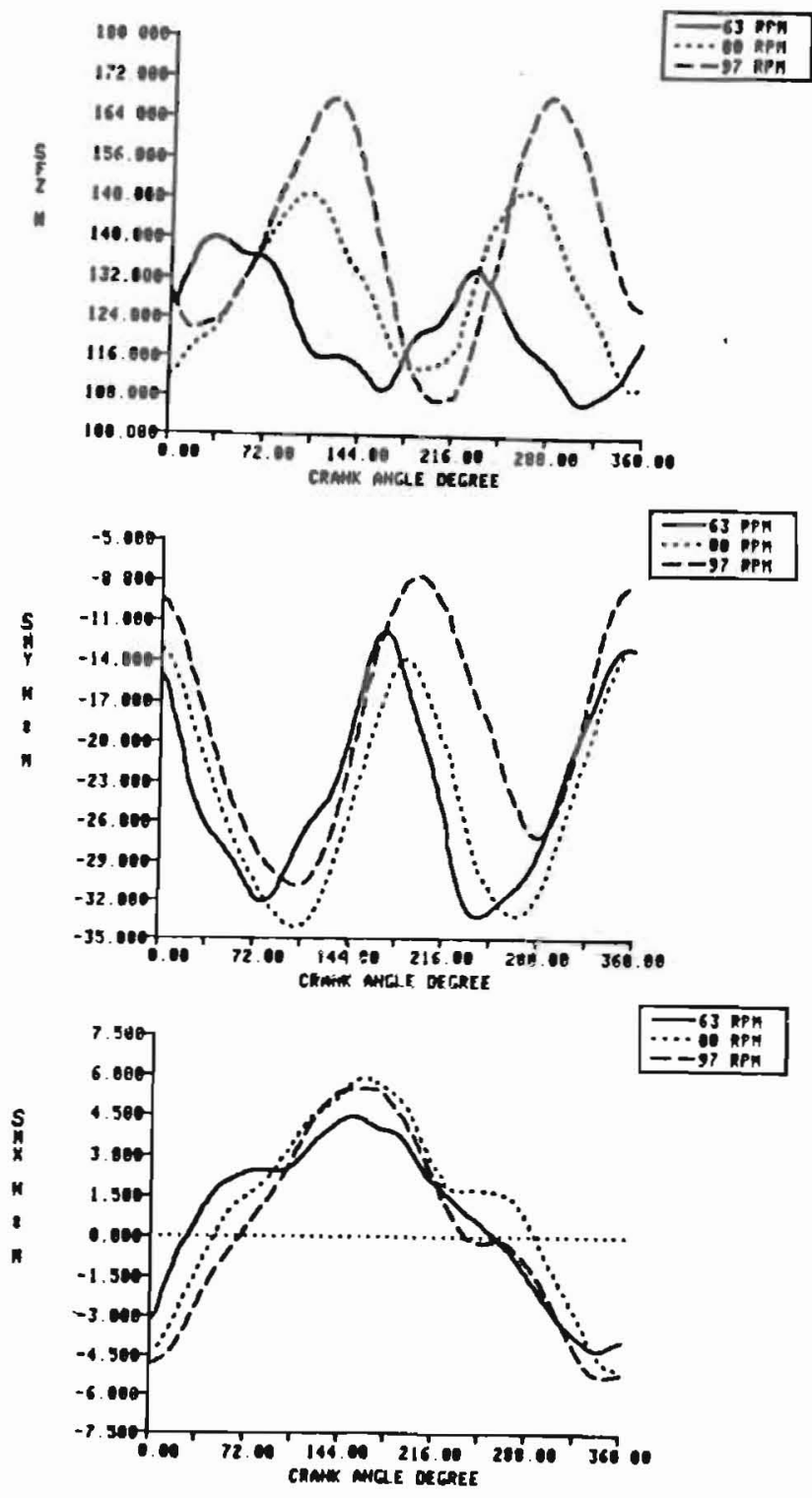

Figure 11. Seat load profiles for rider A 


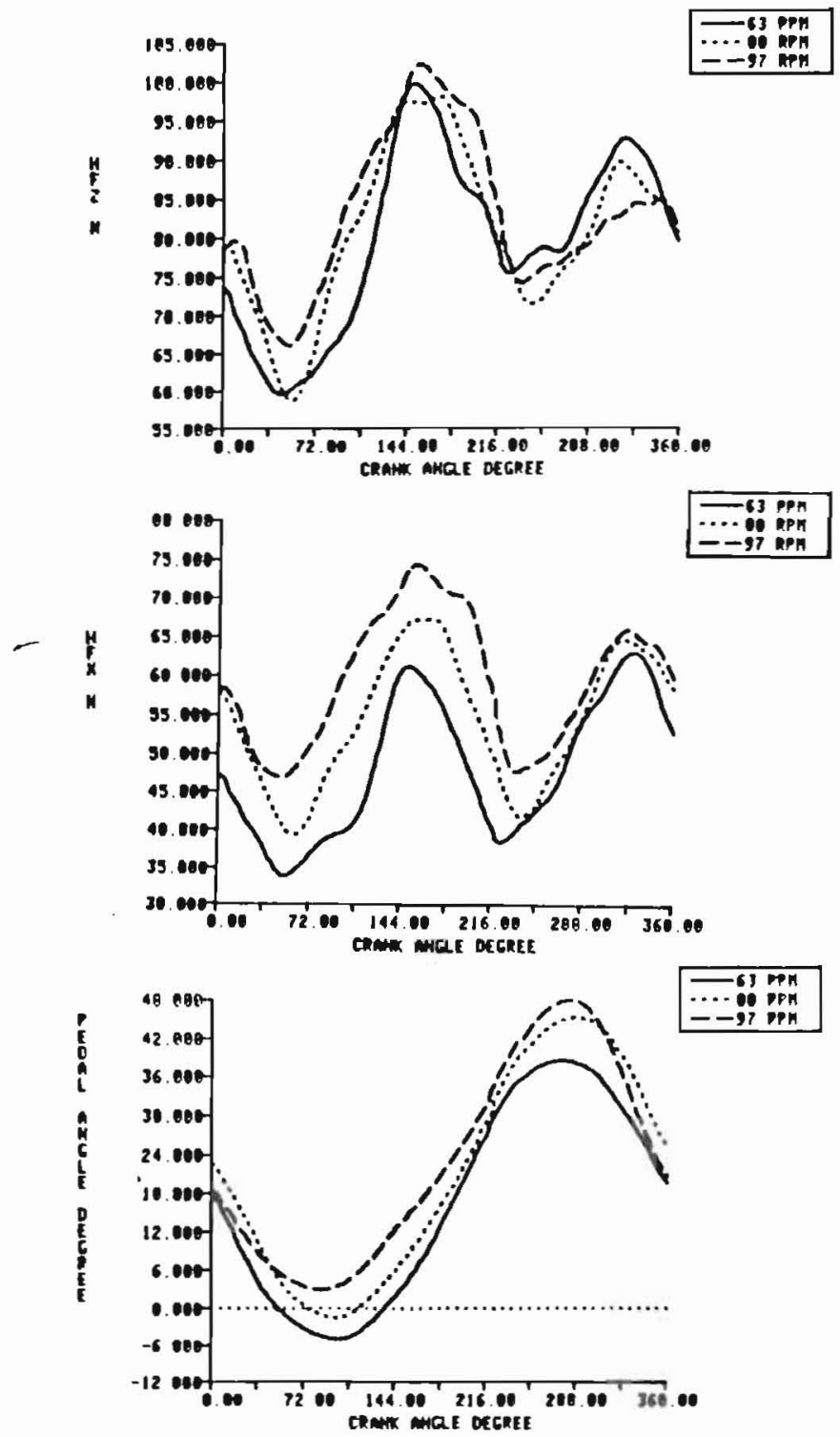

Figure 12. Handlebar load profiles and pedal angle profile for rider $A$ 
surrounding $T D C$. The phase shift that the peak tangential force undergoes may be due to inertia effects which would be expected to be more pronounced at higher RPM.

As illustrated in Fig. 10, increased pedalling rate leads to decreased total torque throughout the entire pedal cycle. The decrease in the positive torque during the downstroke is primarily due to the decrease in the magnitude of the negative normal pedal force. The decrease in the negative total torque during the upstroke, however, is due in part to the increase in magnitude of the negative normal pedal force and in part to the tangential pedal force becoming more positive.

Examining the effects of pedalling rate on both the transverse pedal force PFy and the axial moment $P M z$ emphasizes the relation noted earlier between these two load components and the normal pedal force PFz. The peak negative and positive excursions of PFy and PMz respectively, which occur simultaneously with the peak negative normal force PFz, are both inversely related to the pedalling rate. During the upstroke, however, neither of these load components (PFy or PMz) bears a strong relation to the pedalling rate. Examination of both these plots in Figs. 8 and 9 and those for the other subjects evidenced no relation between any temporal characteristics of either Fy or PMz and pedalling rate.

The remaining load to be discussed is the moment PMx. Although rider A exhibits an apparant inverse relation between the peak positive value of PMx, this relation did not hold for the other subjects. When each of the subjects is considered on a case by case basis, however, a consistent relation between magnitude characteristics and the three pedalline rates exists. Accordingly, it must be concluded the while a general relation between pedalling rate and moment PMx $c$ an not be identified, some relation does exist but its nature is subject dependent.

One final point meriting discussion is that in spite of the weight difference among the riders, a large difference in the magnitude of the driving forces PFx and PFz is not observed. This suggests that the difference in rolling resistance between subjects is small. Rolling resistance RR can be calculated based on an empirical formula given by Whitt, (1971) as follows:

$$
R R / W=0.005+0.15 / p
$$

where $W$ is the subject's weight and $p$ is the tire pressure in units of psi. Calculating $R R$ for riders $A, B$, and $C$ gives $3.76 \mathrm{~N}, 3.56 \mathrm{~N}$, and $4.77 \mathrm{~N}$ respectively. Thus a maximum difference of $1.21 \mathrm{~N}$ exists between riders $B$ and $C$ which is certainly negligible compared to the driving forces.

\section{Handlebar and Seat Loads}

Both handlebar and seat load profiles exhibit features which are independent of both subject and pedalling rate. One feature is that handlebar and seat load profiles (except SMX which will be discussed later) go through two complete periods in every crank cycle (see Figs. 11 and 12). This is due to the fact that in each crank cycle both feet are taking part in driving the bicycle. Because both the handlebar and the seat loads are 
reactive to the pedal loads, it is expected to see them having the two periods.

A second feature concerns SMy which reaches its least negative (or most positive) value at about $0^{\circ}$ and $180^{\circ}$ (see Fig. 11). According to the local coordinate system (see Fig. 5), this suggests that at these positions the point of application of SFz moves towards the front of the seat, thus contributing to this positive change in SMy. This is intuitively consistant because at each of these crank angles either the left or the right foot is at the BDC. As the legs reach maximum extension the point of application of SFz would be expected to move towards the front of the seat.

Contrasted to all other seat and handlebar loads, the lateral seat moment SiMx completes only one cycle in every crank rotation (see Fig. 11). For this load the most negative value occurs at $0^{\circ}$ while the most positive value occurs at $180^{\circ}$. Referring to the local coordinate system in Fig. 5 , it is seen that a negative $\operatorname{SMx}\left(\right.$ at $0^{\circ}$ ) results from the point of application of SFz moving into the paper. This action implies that the right hip is contributing to SFz. This agrees with intuition because at this position the left leg is fully extended so that the seat does not support the left ischial tuberosity. The positive $S M x$ at $180^{\circ}$, which corresponds to full extension of the right 1 eg, results from the seat supporting the left isetial tuberosity.

Similar to the other seat loads, the handlebar forces $\mathrm{HFx}$ and $\mathrm{HFz}$ experience two periods while remaining in phase at all times (see Fig. 12). The correspondence between these forces is not surprising because they are the two components of the force exerted by the hand in the direction of the stretched arm. This is also evident from their relative magnitudes. The ratio $\mathrm{HFz} / \mathrm{HFx}$ is in the range of $1.4-2.1$ depending on the rider which indicates an angle between the resultant force and horizontal of 55 to 65 degrees (see Fig. 4). One final point is that $H F X$ and $H F z$ reach their peak at about $140^{\circ}$ and $320^{\circ}$. As was discussed for SMy, the body is coming back up after $180^{\circ}$. Therefore, it is anticipated that handlebar forces reach their maximum value before this position is reached.

Considering the effect of the RPM on the non-driving forces, it should be expected that the RPM would affect the seat and handlebar forces in an opposite manner to the driving forces on the pedals. Focussing first on the handlebar forces, it is seen that in general Case 3 (high RPM) is the high bound and.Case 1 (low RPM) is the low bound which agrees with the above statement. It can also be seen that the RPM does not affect the phase of these profiles.

RPM has a pronounced effect on the vertical seat force and little effect on the seat moments. Examining SFz (see Fig. 11), it is observed that again Case 3 is the high bound and Case 1 is the low bound. In fact a difference of about $30 \mathrm{~N}$ exists between Cases 1 and 3 which is about 20 percent of the force itself. The RPM not only affects the magnitude of SFz, but it also produces a phase shift of about $20^{\circ}-70^{\circ}$ between Cases 1 and 2 or 3 . The seat moments SMX and SMy, however, are not considerabiy affected by the RPM because they are primarily produced due to the eccentricity of the vertical seat force SFz which is basically independent of the RPM. 
As can be seen in examining all load and angle profiles, all three riders show basically the same Lehavior. Yet due to nature of experimenting with different subjects one would expect some individual characteristics. One individual characteristic concerns the polarity of the seat moment SMy. Contrasted to the data for rider A in Fig. 11, rider B shows a positive SMy which indicates that he is seated more toward the front of the seat compared to rider A. Another individual characteristic is apparent when SFz of different riders are compared against each other. For example a phase differece of about $35^{\circ}$ exists between Case 1 of riders $A$ and $B$. Also, as was mentioned previously, a phase shift exists among different cases of each rider. Therefore it is difficult to generalize a relationship between the profiles of SFz and any other load. For most cases, however, a relationship exists between SFz and SMy. This relationship is that SFz decreases as SMy gains a net positive change (e.g. see Figs. 11 Cases 2 and 3 ). This means that as the point of application of SFz is moved towards the front of the seat, its magnitude decreases. Yet due to the above mentioned phase shifts it is best not to generalize this relationship and consider SFz as force which is affected by both the RPM and the styles of individual riders.

\section{SUMMARY}

\section{A. Pedal Loads}

The pedal forces exhibited characteristics, which can be broadly categorized, as being both subject and pedalling rate independent and subject independent but pedalling rate dependent. One characteristic independent of both variables is that the normal pedal force $\mathrm{PFz}$ is responsible for the majority of torque which reaches a peak at about $100^{\circ}$. Both the transverse pedal force PFy acting outward on the pedal and an axial moment PMy acting internaliy accompany the normal pedal force. Although the normal pedal force PFz is the major contributor to the torque developed on the crank arm in the region around $90^{\circ}$, the tangential pedal force PFx may be a major contributor to the crank torque in the regions around $250^{\circ}$ and $T X$. The PMy moment, which acts about the pedal spindle, is enabled by interaction of the shoe and pedal and serves to equilibrate the moment created by the tangential pedal force PFX. Accordingly PMy tracks PFx except that the polarities are reversed. The only pedal load not exhibiting characteristics in this category is the pedal moment PMx.

Pedalling rate has a consistent and pronounced effect on all pedal loads except the pedal moment PMx. The magnitudes of all loads bear an inverse relation with pedalling rate at constant power. In the case of the driving forces $\mathrm{PFX}$ and $\mathrm{PFz}$ this result follows directly from mechanical considerations. For the other loads, however, this result stems from the relationship between these loads and the driving forces.

\section{B. Handlebar and Seat Loads}

Similar to the pedal loads, both handlebar and seat loads exhibit features falling into the same two categories. Features independent of both subject and pedalling rate will be discussed first. All handlebar and seat loads except SMx exhibit two cycles in each crank cycle. This result holds because handlebar and seat loads are reactive to pedal loads. The seat 
moment SMy exhibits a phase relationship which is consistent with full extension of either leg. At these leg positions the moment takes its greatest value indicating that the point of application of the vertical seat force SFz, which creates the moments, moves forward. Unlike all other seat and handlebar loads, the seat moment SMX goes through one cycle for each crank cycle. Maximum and minimum values coincide with full extension of the right and left legs respectively. The conclusion is that the seat moment SMX is created by the seat supporting the ischial tuberosity opposite to that of the extended leg.

Inasmuchas handlebar and seat loads are reactive to the driving forces on the pedals, pedalling rate has a consistent but opposite effect on the seat and handlebar loads most strongly related to the driving loads. This observation always holds for the handlebar forces and usually the vertical seat force SFz. The seat moments SMy and SMx are not strongly affected by pedalling rate.

\section{ACKNOWLEDGEMENTS}

The authors are grateful to the University of California Appropriate Technology Program (Grant No. 83-14-6000) for supporting this work. We also thank the bicyclists that volunteered their time and energy, S. Wilson, W. Bessom, -and especially L. Halsted who helped during the development stages of the experiment. Finally, we wish to acknowledge Ms. Jane Jandera who very ably prepared the manuscript.

\section{REFERENCES}

1. Davis, R. R. and Hu11, M. L., "Measurement of Foot-Pedal Loads During Bicycling: Data Analysis and Results", Journal of Biomechanics, Vol. 14, No. 12, 1981b, pp. 857-872.

2. Gregor, R. J., "A Biomechanical Analysis of Lower Limb Action During Cycling at Four DIfferent Loads", Ph.D. Thesis, Physical Education, Pennsylvania State University, 1976.

3. Hoes, J. J. A. J. M., Binkhorst, R. A., Smeekes-Kuyl, A. E. M. C., Vissers, A. C. A., (1968), "Measurement of Forces Exerted on Pedal and Crank During Work on a Bicycle Ergometer at Different Loads," Int. Z. Angew. Physiol. Einschl. Arbeitsphisiol., Vol. 26, pp. 33-42.

4. Hu11, M. L. and Davis, R. R., "Measurement of Foot-Pedal Loads During Bicycling: Instrumentation," Journal of Biomechanics, Vol. 14, No. 12, 1981 a, pp. 843-855.

5. Soden, P. D., and Adeyefa, B. A., "Forces Applied to a Bicycle During Norma 1 Cycling", Journal of Biomechanics, Vol. 12, No. 7, 1979, pp. 527-541.

6. Whitt, F. R., "Estimation of the Energy Expenditure of Sporting Cyclists", Ergonomics, Vol. 14, No. 3, May 1971, pp. 419-429. 